\title{
Le Père de la Brosse
}

Sa vie, son oeuvre

\section{Yves Tremblay}

Volume 35, 1968

URI : https://id.erudit.org/iderudit/1007306ar

DOI : https://doi.org/10.7202/1007306ar

Aller au sommaire du numéro

Éditeur(s)

Les Éditions Historia Ecclesiæ Catholicæ Canadensis Inc.

ISSN

0318-6172 (imprimé)

1927-7067 (numérique)

Découvrir la revue

Citer cet article

Tremblay, Y. (1968). Le Père de la Brosse : sa vie, son oeuvre. Sessions d'étude Société canadienne d'histoire de l'Église catholique, 35, 47-59.

https://doi.org/10.7202/1007306ar

Tous droits réservés @ Les Éditions Historia Ecclesiæ Catholicæ Canadensis Inc., 1968
Ce document est protégé par la loi sur le droit d'auteur. L'utilisation des services d'Érudit (y compris la reproduction) est assujettie à sa politique d'utilisation que vous pouvez consulter en ligne.

https://apropos.erudit.org/fr/usagers/politique-dutilisation/ 


\section{Le Père de la Brosse}

\section{SA VIE, SON CEUVRE}

Retracer en quelque vingt minutes la vie d'un homme, surtout une vie aussi dense et aussi riche que celle du Père de la Brosse, jésuite, oblige forcément à ne souligner et d'une manière bien expéditive que quelques aspects de son existence et de son œuvre. C'est pourquoi, la première partie de cet entretien aura l'aspect d'une chronologie et la seconde, celui d'une bibliographie sommairement commentée.

\section{- I -}

Le Père de la Brosse naquit le 30 avril $1724^{1}$, à Magnat, hameau de la paroisse de Jauldes, dans l'Angoumois, aujourd'hui diocèse d'Angoulême, du mariage de Jean de la Brosse, sieur de Chabrouillière et de Louise Duboiscuvier. Il fut baptisé, dit-on, le lendemain dans l'église de Saint-Martin de Jauldes, par le curé de cette paroisse, un monsieur de la Brosse, archiprêtre, vraisemblablement un parent du nouveau-né. Sa marraine, madame Jeanne Mayon, était une hospitalière de la Rochefoucauld ${ }^{2}$. Cet enfant, le septième, appartenait à un milieu qui depuis des siècles donnait des prêtres à l'Église. Il n'avait que huit mois lorsque son père décéda. Si l'épreuve fut douloureuse, la maman ne manqua pas de zèle, car grâce à sa vaillance et à la collaboration de maitres choisis, le jeune Jean-Baptiste reçut une éducation soignée et toute l'attention nécessaire pour développer ses talents.

Après avoir fait sa première communion à Jauldes ${ }^{3}$, il entre au collège Saint-Louis-d'Angoulême dirigé par les pères jésuites, au commencement d'octobre 1735 , et il y reste jusqu'à la fin de sa réthorique en juillet 1740. Deux mois plus tard, le neuf octobre 1740, on le retrouve au noviciat des Jésuites de Bordeaux ${ }^{4}$. Il fut reçu par le père Nectroux, recteur de cette maison. Après deux années de formation, le Père de la Brosse prononça ses premiers vœux à la messe dite par le père Nectroux, dans la chapelle domestique du noviciat, le 10 octobre $1742^{5}$.

1 Date qu'il donne lors de son entrée au noviciat de la Compagnie de Jésus (P. C. Rochemonteix, s.j., Les Jésuites et la Nouvelle-France au $\mathrm{VR}^{e}$ siècle, p. 217.)

Libérale, 26 avril 1889 , p. 3.

3 A. Chambre, Le R. P. J.-B. de la Brosse, p. 158.

4 P. C. Rochemonteix, s.j., op. cit., p. 217, 218.

5 A. E. J. Loc. cit. 
Le noviciat terminé, il fait deux ans de philosophie à Pau (1742-44), puis il enseigne à Pau et à Bordeaux toutes les classes à partir de la cinquième jusqu'à la rhétorique inclusivement (1744-49) . Pour répondre au exigences de la formation que requiert la Compagnie de Jésus, il laisse l'enseignement et se consacre de nouveau pendant un an à l'étude de la philosophie (1749-50). Suivront immédiatement après. quatre années d'études en Théologie (1750-54). Il sera ordonné prêtre après trois ans de théologie, soit le deux février 1753. Ảu terme de ses études, âgé de $\widehat{2} \hat{y}$ ans, le Père de la Drosse esi vuué à une carrière apostolique des plus fructueuses et ses supérieurs le destinent aux missions du Canada.

Par son passage en terre canadienne, le Père de la Brosse était rangé parmi les membres de la Compagnie de Jésus, de la Province de France à laquelle la mission du Canada était échue. Son nom est porté pour la première fois, en 1754, dans les catalogues de la province de France comme missionnaire au Canada, mais sans mission encore déterminée, c'est-à-dire. sous le titre général "apud varias gentes ». Vraisemblablement, il aurait passé la première année à Québec, occupé aux divers travaux du ministère pastoral.

Sa première obédience en terre canadienne, l'assigne comme missionnaire à la Rivière Saint-Jean, dans le Nouveau-Brunswick actuel. chez les sauvages Abénaquis. Tout nous laisse croire cependant, qu'il s'occupa davantage des Acadiens que des Abénaquis. Nous en sommes davantage convaincus, quand nous lisons dans une lettre de l'abbé le Guerne à l'abbé de l'Isle Dieu, le fait suivant: "Le Père de la Brosse vint à Petcondiac et me débarassa (sic) de cette rivière et de Chipoudy jusqu'à Pâques auquel il s'en retourna à Québec ${ }^{6}$." Dans la même lettre, le nom du Père de la Brosse revient quand l'abbé le Guerne décrit à son ami, les efforts qu'il fait pour soustraire les Acadiens à la merci des Anglais: "Je leur marquois fort au long (d'une lettre) tous les dangers qu'ils couraient à rester dans leurs quartiers et que le père labrosse (sic) leur expliqua de ma part ${ }^{7}$." Les registres de Petcondiac et de Chipoudy sont encore plus révéla. teurs. "En effet, dans un acte du 16 juin 1756, consigné dans les registres, le Père de la Brosse atteste que les actes qui ont été faits du 16 novembre 1755 au 16 mars 1756, sont authentiques et sont insérés dans les registres de Petcondiac et Chipoudy. " Et il ajoute qu' i il a exercé les fonctions curiales dans ces endroits par la permission de Guerne, curé de l'Acadie et Vicaire-Général de Mgr. de Québec ${ }^{8}$. Il n'a pas non plus quitté l'Acadie à Pâques, car l'acte auquel nous

p. 43.

6 C. O. Gagnon, Lettre de M. l'Abbé le Guerne, missionnaire de l'Acadie,

7 Idem., p. 46.

8 Idem., p. 13. 
venons de référer est daté du 16 juin 1756. Il semblerait plutôt qu'il regagne la Rivière Saint-Jean à Pâques et qu'il revient de temps en temps faire du ministère à Petcondiac et à Chipoudy. Il réside encore à la Rivière Saint-Jean le 31 juillet 1756, car il exerce les fonctions de secrétaire pour un groupe d'Acadiens arrivés au Port Saint-Jean au début de janvier 1756. Ceux-ci adressent une lettre à l'abbé Daudin, dernier curé de Port-Royal ${ }^{9}$. Par la suite, le Père Germain jugeant sa présence inutile auprès des Indiens, le Père de la Brosse aurait laissé la Rivière Saint-Jean à la fin de l'été ${ }^{10}$.

A son retour à Québec, le Père de la Brosse sera procureur, économe, consulteur du collège et professeur de philosophie. Les archives du séminaire de Québec conservent un précieux souvenir de son professorat. Il s'agit des notes de cours d'un de ses élèves, André Couillard 11. A la page huit, on peut lire cette présentation: "Cours de philosophie, donné par le Père de la Brosse au collège de Québec, 1757. Philosophie, première partie, logique." Ce manuscrit de 421 pages est tout à la gloire de l'élève du Père de la Brosse. L'écriture est soignée et même des dessins à la plume ou mieux des tableaux synthèses signalent les diverses parties. On retrouve à la fin un tableau résumant les formes de syllogismes.

A l'automne de la même année, l'abbé Récher, curé de Québec, rapporte dans son journal, le fait suivant au sujet du Père de la Brosse: « 18 septembre 1757, mort du Père Gélase, Récollet, qui a été enterré aux Récollets, y ayant été apporté sur un cabrouet, le Père de Labrosse, jésuite, le précédant en étole avec la croix et deux acolytes, jusqu'à la porte des Récollets, ce qui n'a pas été approuvé ${ }^{12}$. "Une question de formalités aurait occasionné cette désapprobation.

L'année 1758 est une année mémorable dans la vie spirituelle du Père de la Brosse. En effet, il entre en retraite le soir du 24, janvier pour se préparer au grand événement de sa vie à cette époque et mercredi matin, le deux février, fête de la Purification, il fait publiquement dans l'église du collège, la profession des quatre vœux, entre les mains du Père Claude Godefroy Coquart, en cette occasion, député du Père de St-Pé, supérieur de la mission et recteur du Collège, alors dans sa $72^{e}$ année et souvent incapable, à cause de sa faiblesse, de présider à de semblables cérémonies ${ }^{13}$. Quelques jours après cet

p. 50,51 .

9 P. Gaudet, Une épisode de l'expulsion des Acadiens dans BRH, vol. XIV,

10 P. C. Rochemonteix, s.j., op. cit., p. 219.

11 Arch. du Séminaire de Québec, Manuscrit série $M, n^{\circ} 67$.

$12 \mathrm{M}^{\mathrm{gr}}$ TÊTU, M. Jean-Félix Récher, curé de Québec et son journal, 1757-1760 dans $B R H$, vol. IX, p. 129.

13 Arch. Jés. de Saint-Jérôme, autrefois Arch. Coll. S.-M., Dioc. 673. 
événement, il deviendra chapelain de l'Hôpital Général. Il occupera ce poste pendant deux mois, soit du 15 février 1758 au 3 avril ${ }^{14}$.

Bien avant que la guerre n'établisse l'état de siège devant Québec. le Père de la Brosse aura quitté la ville pour exercer son ministère à Yamaska. Il est assigné à ce poste depuis l'automne de 1758 et y demeurera au moins jusqu'au six juin 1759, date de la signature de son dernier acte. Après deux mois d'absence, il revient à Yamaska où il signe les registres à partir du six août jusqu'au 26 octobre $1759^{15^{2}}$.

Que devient-il pendant ces deux mois d'absence? Il faut se rappeler que nous sommes pendant le conflit de 1759 et que les événements se précipitent à mesure que la tension monte. Nous savons que le Père de la Brosse est venu d'Yamaska avec ses sauvages et qu'il se fait capturer à la Pointe-aux-Trembles (Neuville) le 21 juillet avec 200 femmes, lors d'une descente-éclair des Anglais à cet endroit ${ }^{15^{\mathrm{b}}}$. Amené sur leurs vaisseaux avec les autres prisonniers, il sera relâché le lendemain, près de Québec, car d'après le journal de l'abbé Récher. nous apprenons que les femmes et les enfants qui avaient été capturés à Neuville, furent tous débarqués le lendemain à Sillery vers cinq heures du soir ${ }^{16}$.

Entre le six juin et le 21 juillet 1759, le Père de la Brosse a sûrement passé quelque temps à Québec, comme en fait foi le journal de l'abhé Récher: "J'apprends que monsieur Wolfe, général anglais, a trouvé dans la cassette du Père de Labrosse, deux lettres de moi: l'une que J'écrivais à Mgr à Charlesbourg; et l'autre que j'écrivais au Père Marsolet, récollet aux Trois-Rivières ${ }^{1 \top}$. " Le général Wolfe a donc mis la main sur la cassette du Père de la Brosse, lors de sa capture à Neuville, il n'y a pas de doute. Ce qui reste obscur c'est la présence de la lettre de l'évêque dans cette cassette. Quand cette lettre fut-elle remise au Père de la Brosse? Pourquoi n'est-il pas allé à Charlesbourg avant de retourner à Neuville et pour quelle raison est-il à Neuville ce jour-là ? Malheureusement le document que nous avons en mains ne nous a pas permis d'éclaircir ce fait. Quant à la lettre destinée au Père Marsolet, ça ne pose pas de problème, car il est normal que le Père de la Brosse passe par Trois-Rivières avant de se rendre à Yamaska. On peut donc croire, sans pouvoir le prouver exactement, que le p. 100 .

14 P. G. Roy, Le Père de la Brosse et Montcalm dans BRH, vol. XLII.

$15^{a}$ M. TrudeL, Que devient le Jésuite de la Brosse au moment de la con. quête dans $B R H$, vol. LIX, p. 195-199.

$15^{\text {b }}$ Ibidem.

16 L'abbé Jean-FÉLIx RÉcher, Journal du Siège de Québec en 1759, Cahier $\mathrm{n}^{\circ}$ XI, La Société historique de Québec, 1959, p. 22, 23.

17 Ibidem. 
Père de la Brosse a séjourné à Québec, avant et après sa capture par les Anglais.

Après la capitulation de Québec, le Père de la Brosse est attaché à la maison des Jésuites de Montréal. C'est dans la région de Terrebonne qu'il exercera son ministère car son " nom apparaît fréquemment dans les registres en 1760 , sans que nous puissions retracer sa présence ailleurs ${ }^{18}$ ». Enfin, le 14 mai 1761, "sur l'ordre du grand vicaire Montgolfier, il commence à assurer d'une façon régulière et pour longtemps la desserte de Saint-Henri-de-Mascouche: il en signe les actes jusqu'en $1766^{19}$ ». Le Père de la Brosse a signé son premier acte le 13 septembre 1761 et son dernier le 12 juin 1766 . Pendant les cinq années, comprises entre ces dates, il a signé plus de 450 fois, plusieurs fois chaque mois de chaque année ${ }^{20}$. Il se rend à l'occasion aider le Père Coquart en bas de Québec ${ }^{21}$.

A partir de 1766, jusqu'à sa mort, soit pendant 16 ans, il se dépensera sans compter pour l'évangélisation et l'instruction des Montagnais et assurera également les services de la pastorale auprès des Blancs.

Dans la réponse de $\mathrm{Mgr}$ Briand à une lettre de M. St-Luc La Corne qui réclamait qu'on rende le Père de la Brosse aux habitants de Mascouche, on peut constater l'étendue géographique du champ d'apostolat du Père de la Brosse: "Il (le Père de la Brosse) est chargé de plus de 40 lieux, depuis les îles Jérémie, les Sept-Iles, les MilleVaches, Chicoutimi, Tadoussac, la Malbaie, les Éboulements et l'Ilesaux-Coudres et en outre les sauvages Montagnais. Voilà, monsieur, une province pour l'étendue, remplie de postes où il $\mathrm{y}$ a des catholiques ${ }^{22}$. » A ce territoire, déjà trop vaste, à la mort du Père Ambroise Rouillard, récollet, survenue en 1771 , le Père de la Brosse devra ajouter la desserte des Acadiens de Cacouna, des Français du voisinage de l'IleVerte, de Sainte-Marie-des-Anges et de Saint-Jean-Baptiste sans oublier celle des Acadiens de la Baie des Chaleurs et des sauvages micmacs de Restigouche.

Dans la vie de l'Abbé Bourg, premier prêtre acadien, l'abbé Arthur Melançon souligne cet événement.

Les Acadiens déjà assez nombreux à la Baie des Chaleurs furent privés de tout secours religieux jusqu'à l'arrivée en 1771 du Père de la

18 M. TrudeL, L'Eglise canadienne sous le Régime Militaire, t. II, p. 137n.

19 M. TRUdel, Que devient le Jésuite de la Brosse au moment de la conquête dans $B R H$, vol. LIX, p. 195-198.

Sém. de Québec, fonds Sém., carton 14, liasse 7, $\mathrm{n}^{\circ} 29$.

20 Arch. Jés. de Saint-Jérôme autrefois Arch. Coll. S.-M., Doc. 674, p. 9.

21 M. Trudel, op. cit., p. 195-198.

22 Réponse de $M^{\text {or }}$ Briand à M. St-Luc La Corne, 27 sept. 1763, Arch. du Sém. de Québec, fonds Sém., carton 14, liasse 7, nº 29. 
Brosse, le célèbre missionnaire jésuite du Golfe Saint-Laurent, qui à cette époque vint y faire une courte apparition. Il choisit Bonaventure pour sa résidence, visita Tracadièche, où il bâtit une chapelle, la première élevée dans cette région de la baie; à l'automne de la même année, il était à Ristigouche et bénissait la nouvelle église des sauvages récemment construite sur la rive nord de la rivière, du côté de Québec. Le Père de la Brosse poussa ensuite ses visites jusqu'à la rivière Saint-Jean ${ }^{23}$.

De ce vaste territoire, Tadoussac restera le centre même si le Père de la Brosse n'y réside pas continuellement.

L'emploi du temps varie selon les saisons. En hiver, il apprend à lire et à écrire aux sauvages. Il leur enseigne même à chanter selon la note et à servir aux cérémonies religieuses. Parfois, des jeunes gens, désireux de s'instruire, partagent avec lui ces longs hivernements. C'est ce que nous révèlent ses annales ${ }^{24}$ et une lettre adressée de l'Ile-Verte à une dame de Rimouski, le 23 septembre $1776^{25}$.

Selon les besoins et le bénéfice de son apostolat, il hivernera tour à tour de 1766 à 1776, quatre fois à Tadoussac, deux fois à l'Isle Verte. une fois à Bersiamis en bas des Islets-de-Jérémie, une fois à SaintLaurent Ile d'Orléans, où il travaille à son dictionnaire montagnais. une fois à Bonne-Aventure et une fois à Chicoutimi. A partir de 1776, jusqu'à sa mort, les annales du Père de la Brosse n'ont pas été retrouvées et on ne peut établir les différentes étapes de ses pérégrinations. Il continue à exercer son ministère aux mêmes endroits comme en fait preuve un acte du gouverneur Haldimand, daté du deux mai 1781 dans lequel il accorde au Père de la Brosse la permission d'exercer les fonctions de son ministère " en dedans du fleuve et du Golfe Saint-Laurent, pour les Sauvages, les Canadiens et les Acadiens résidant dans ces lieux ${ }^{26}$ ".

La mort viendra mettre un terme à ses travaux apostoliques, le 10 avril 1782. Il sera assisté dans ses derniers moments par l'abbé Compain, curé de l'Ile-aux-Coudres. Son corps sera inhumé dans la chapelle de Tadoussac, au pied des marches de l'autel où il repose toujours.

$$
\text { - II - }
$$

Le Père de la Brosse savait qu'il ne pouvait demeurer toujours auprès des Montagnais. Il était conscient d'autre part, que la présence permanente d'un missionnaire dans les Postes du Domaine du Roi,

23 L'abbé Arthur Melançon, Vie de l'ablé Bourg, premier prêtre acadien canadien, p. 56, 57.

24 Notes du Père de la Brosse sur ses missions dans Histoire du Saguenay de l'origine jusqu'à 1870, p. 143.

25 R. G. ThwaItes, The Jesuit Relations and Allied Documents, Vol. LXXI, $\mathrm{n}^{\mathrm{C}} 20 . \mathrm{n} .310$.

26 Arch. acad. de l'Univ. de Moncton, Papiers de Placide Gaudet, boite 53. 
après son départ, vu les faibles effectifs du clergé canadien, serait un fait presque irréalisable. Pour assurer alors une certaine permanence à son œuvre, le Père de la Brosse composa de nombreux travaux en langue montagnaise. Certains nous sont parvenus sous forme d'imprimés, d'autres sous forme de manuscrits. Considérons d'abord les ouvrages imprimés.

Dans les cahiers de comptes des imprimeurs Brown et Gilmor de Québec, nous découvrons la liste des travaux que le Père de la Brosse leur a confiés. Cependant, l'imprimeur n'abuse pas de mots et son laconisme est loin de satisfaire notre curiosité.

Dès 1766, en octobre, le Père de la Brosse fait imprimer 1,000 calendriers. La coutume de distribuer des calendriers aux Indiens existait depuis assez longtemps. La Relation de 1640, nous donne la raison d'être de ce calendrier, sa composition et l'usage que les Indiens en faisaient:

Comme ils (les Indiens) devaient être longtemps (éloignés) nous donnâmes aux chrétiens un calendrier pour reconnaître les dimanches afin de faire leurs prières un petit peu longues ces jours-là; or comme ils ne savaient ni lire, ni écrire, on avait distingué les jours, les lunes et les fêtes par diverses marques, leur donnant ce papier comme à l'aventure, pour voir s'ils s'en pourraient servir. Je vous assure que nous fûmes bien étonnés à leur retour, car nous étant venus voir, après avoir remercié Dieu en la chapelle, ils nous apportèrent leurs papiers et nous dirent : . Voyez si nous ne nous sommes pas mécomptés, voilà le jour où nous pensons être , , firent-ils, ils ne s'étaient pas mépris d'un seul jour, \& voilà, ajoutaient-ils, les jours de dimanche, nous les avons gardés tous, excepté celui-là, qu'ils montraient, nous l'avons marqué exprès pour vous le montrer. Nous dîmes qu'il fallait vous en avertir: le dégel nous contraignit de travailler ce jour-là, nous étions marris, mais nous étions en danger de perdre nos provisions; les jours de fêtes, nous nous assemblions et priions Dieu dans une cabane et nous chantions ce que nous savions, les autres jours chacun priait chez-soi ${ }^{27}$. ,

L'expérience était concluante et ce moyen d'apostolat répondait aux besoins du temps. Il est fort probable cependant, que les calendriers imprimés du Père de la Brosse, différaient des calendriers indiens de 1640, car les Montagnais savaient déjà lire. Nous pouvons croire que le Père de la Brosse leur avait donné une touche personnelle et qu'ils devaient sensiblement ressembler aux nôtres. L'imprimeur inscrit la remarque suivante dans son cahier:

Kalendrier perpétuel à l'usage des sauvages montagnais, mistassins, papinachois et autres des Postes du Roi, de Tadoussac, Chicoutimy, Rivière de l'Assomption, etc. 28

27 R. G. Thwaites, op. cit., vol. XVIII, p. 166.

28 M. Tremaine, A Bibliography of Canadian Imprints, 1751-1800, p. 36. 
Une annonce parue dans la Gazette de Québec du 20 octobre 1766 précise que "ce Kalendrier veut servir à tous les autres sauvages qui vont en chasse ou qui passent un tems (sic) considérable loin du lieu de la mission 29 ". Le cahier de l'imprimeur Brown nous informe que le Père de la Brosse fit de nouveau imprimer, en mai 1768, 199 calendriers. En septembre 1769, il passe la commande pour quatre ans. En 1773, l'imprimeur s'engage à faire 127 exemplaires de ce calendrier pour chacune des années de 1773 à 1778. Enfin, une dernière

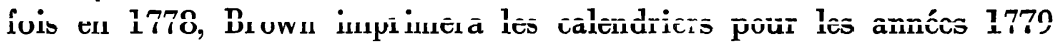
à 1785,500 copies par année. Il ne reste aucune copie de ces calendriers qui devaient se résumer à quelques feuilles.

Pour aider les Montagnais qui savaient déjà lire et leur permettre d'enseigner à leurs congénères, le Père de la Brosse fit imprimer en 1767 un alphabet en montagnais. C'est un petit opuscule de huit pages, sans page-titre. le texte commençant dès la première page. Entièrement écrit en montagnais, il renferme l'alphabet, des syllabes, des prières et autres formules de dévotion. Le seul exemplaire qui a pu être retracé est conservé aux archives de l'archevêché de Québec.

En 1767, également, le Père de la Brosse fait imprimer un Catalogue des Indiens de Tadoussac. L'imprimeur ne donne aucun détail concernant cet ouvrage et aucune des 200 copies ne peut être retracée.

Nous pouvons entrevoir l'utilité de ce catalogue et sa raison d'être dans la note que le Père de la Brosse a inscrite au début du manuscrit intitulé Magnus Liber. Ce manuscrit a été commencé par le Père Coquart et il devait contenir selon un ordre déterminé les baptêmes, les mariages et les sépultures. Mais la méthode ne fut guère suivie et le désordre qu'il renferme semble fatiguer le Père de la Brosse comme nous le révèle la note qu'il écrit au début du manuscrit :

Outre que dès la quatrième page, les Mariages sont confondus avec les Baptêmes, dans la plupart des actes les personnes sont nommées par le seul nom de Baptême, ce qui fait une confusion et un cahos impénétrables, parce que les mêmes noms sont donnés à plusieurs personnes au Baptême.

Non sans peine on est venu à bout d'ôter une partie de cette confusion en évitant le concours des mêmes noms de Baptême et de famille, ce qui est aisé à continuer en observant de consulter les tables alphabétiques des noms de la nation 30 .

Il semble bien que ces tables alphabétiques, et le catalogue sont une seule et même chose et vu le nombre d'exemplaires, le Père de la Brosse a dû en faire circuler parmi les familles indiennes.

29 Ibidem.

30 Ce manuscrit est conservé aux archives de l'Archevêché de Québec. 
A l'automne de 1767 , le Père de la Brosse remet à l'imprimeur ce qui nous apparaît la pièce capitale de ses écrits, le livre de prières en montagnais intitulé Nehiro Iriniui-Aiamiche-massinahigan, c'est-à-

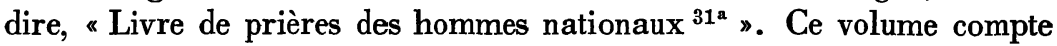
96 pages se distribuant ainsi : page un, le titre; la page deux reste blanche; les pages de trois à cinq renferment l'approbation de Mgr Briand; la page six est blanche; des pages sept à 93 , on peut lire les prières que renferme cet ouvrage et certaines notions de catéchisme; les pages 93 à 96 présentent l'index et la page 96 affiche le colophon et les errata. Le livre est tiré à 2,000 copies et destiné à l'usage des Montagnais du Saguenay, du Lac Saint-Jean et autres, comme l'indique la page-titre. Le nom de l'auteur apparaît en montagnais Jean-Baptiste Tshitshisahigan, c'est-à-dire, la brosse.

La traduction des prières catholiques en montagnais ne se fait pas sans difficultés et sans donner lieu à une gymnastique intellectuelle assez subtile. La langue montagnaise ne se prête pas facilement à la transcription des formules telles que nous les connaissons et les mots montagnais adoptent difficilement la résonance et la signification que nous donnons à certains mots familiers que contiennent nos formules de prières. Ainsi la première demande du Pater, de l'ancienne formule, "Notre Père, qui êtes aux cieux, que votre nom soit sanctifié ", est rendue par le Père de la Brosse en montagnais par la formule suivante: "Notre Père, toi dans le Ciel qui es. Il fait grand ton nom ${ }^{31^{b}}$."

Si nous voulions pousser plus loin dans la même veine, on constaterait que la formule du Père de la Brosse varie de celle du Père Massé, qui fut le premier à traduire le Pater en montagnais ${ }^{31 \mathrm{c}}$. Il faut donc reconnaître une certaine évolution linguistique qui impose des formules nouvelles sans pour cela aplanir les difficultés d'une traduction adaptée aux Indiens ${ }^{31^{d}}$.

Ce volume fut réimprimé à Québec en 1817 et en 1844 .

Le 13 mai, 1769, Mgr Briand publie un mandement à l'intention des « Montagnais de Tadoussak, des Islets de Jérémie, de SaintNicholas, des Sept Iles, de Shekoutimi, des Lacs Saint-Jean et Shononskouan, de Portneuf, et à toute la Nation quelque part qu'elle habite ${ }^{32}$ ». Si l'évêque se réjouit du fait que plusieurs Indiens s'appli-

31 N. E. Dionne, Les langues sauvages du Canada dans Congrès international des Américanistes, $\mathrm{XV}^{\bullet}$ session, vol. II, p. 213.

$31^{\mathrm{b}}$ Ibidem.

31 " Notre Père qui es ès Cieux, Ton nom soit en estime... ", traduction de la première demande du Pater par le $P$. Massé, 250 ans avant la traduction du P. de la Brosse, Ibidem.

$31^{\mathrm{a}}$ Idem, p. 214.

32 Mandement de $M^{\text {or }}$ Briand dans L'Oiseau-Mouche, vol. I, $\mathrm{n}^{\circ}$ 5, 25 février 1893. 
quent à lire et à écrire et s'efforcent de l'enseigner aux autres pendant l'absence du missionnaire, il n'est pas, d'autre part, sans s'attrister devant le fait que des Indiens négligent de s'instruire et de vivre conformément à ce qu'enseigne la religion. Il met les Indiens en garde contre les mauvais exemples que peuvent leur donner les Blancs et rappelle à ces derniers les peines qu'ils encourent en se permettant d'enivrer les sauvages. Enfin, il s'adresse aux missionnaires, leur enjoignant de déployer tout leur zèle pour arrêter ce désordre.

$\mathrm{Si}$ le Père de la Brosse fait imprimer ce mandement en montagnais et en français, c'est pour répondre à la volonté de l'évêque a que le présent Mandement soit traduit en langue Montagnaise, lu, publié et affiché dans toutes les églises de la Mission, à la diligence du Missionnaire à qui nous permettons de le faire imprimer, voulant qu'il en renouvelle la lecture toutes les fois qu'il le jugera nécessaire ${ }^{33}$ ".

Enfin mentionnons un dernier ouvrage imprimé en 1770, l'alphabet en abénaquis. Toujours selon le livre de comptes de Brown et Gilmore, encore à l'automne, le Père de la Brosse fait imprimer 600 alphabets indiens en abénaquis. Cet opuscule en dialecte abénaquis est le premier et probablement le seul ouvrage imprimé au Canada dans ce dialecte, au XVIII ${ }^{e}$ siècle ${ }^{34}$. Ce geste de la part du Père de la Brosse nous laisse un peu perplexe. A cette date, le missionnaire ouvre depuis 1766 dans la région du Saguenay et du côté de Sept-Îles auprès des Montagnais. En 1770, il hiverne à l'l̂le-d'Orléans où il travaille à son dictionnaire et nulle part, il ne fait allusion à cet ouvrage. Pour justifier cet imprimé en abénaquis dans les œuvres du Père de la Brosse, nous sommes portés à croire qu'il a fait imprimer cet alphabet pour un autre missionnaire, en l'occurrence, le Père Germain avec qui il avait été missionnaire à la Rivière Saint-Jean et qui fut le dernier jésuite auprès des Abénaquis. Ce dernier d'ailleurs, mourut en 1779 à Saint-François-du-Lac. Nous convenons aisément que c'est une preuve assez sommaire. La bibliothèque de Saint-Sulpice de Montréal garde un exemplaire de cet alphabet ${ }^{35}$.

Le Père de la Brosse a laissé encore d'autres travaux demeurés sous forme de manuscrit. Les Pères Oblats de Marie-Immaculée conservent dans leurs archives du Scolasticat Saint-Joseph d'Ottawa quelques-uns de ses principaux manuscrits.

Signalons d'abord un Dictionnaire montagnais-latin en deux volumes. Le premier tome va de la lettre " $A$ » à « PIR ». Sur la première page, on peut lire en latin une présentation dont la traduction se lirait ainsi :

33 Ibidem.

34 M. Tremaine, op. cit., p. 105.

35 Ibidem. 
Dictionnaire des racines montagnaises, colligé d'après les écrits variés des différents missionnaires, mis en ordre alphabétique par Jean-Baptiste de la Brosse, prêtre de la Compagnie de Jésus, qui fut le $21^{\bullet}$ Père en Dieu et Pasteur des Sauvages montagnais dans les postes du Domaine du Roi.

Ouvrage commencé à Sainte-Anne de Tadoussac, le sixième jour des ides de novembre (huit novembre), année 1766 , à partir de la maternité de la Vierge.

A la fin de ce premier tome, l'auteur donne le nombre de mots correspondant à chaque lettre de " $A$ » à $\propto P$ », et fait le total, ce qui donne 6,546 mots. Le deuxième tome ne comporte aucune présentation et renferme les mots répondant aux lettres \& PIR 》 à \&UUT " et nous retrouvons à la fin, une compilation indiquant pour chaque lettre de « $P$ » à " $U$ " le nombre de mots inscrits. Le deuxième tome renferme 5,245 mots. L'auteur a soin de faire la somme des mots contenus dans les deux tomes et totalise 11,791 mots. Sur la couverture de parchemin, nous pouvons lire le titre écrit à la main et des précisions propres à chacun des deux tomes.

En 1772, le Père de la Brosse reprenait le même ouvrage, mais cette fois allant du latin au montagnais. Ce dictionnaire comprend également deux tomes. Le premier va de " $A$ » à « POM » et l'auteur en fait une présentation suivante en latin dont voici la traduction :

Lexique correspondant au dictionnaire des racines montagnaises, colligé des écrits divers des différents missionnaires, mis en ordre alphabétique par Jean-Baptiste de la Brosse de la Compagnie de Jésus, $21^{\bullet}$ Père en Dieu et Pasteur des Sauvages montagnais dans les postes du Domaine du Roi.

Commencé aux Iles Jérémie, le $14^{\ominus}$ jour des calendes de juillet (18 juin), année 1772 à partir de la maternité de la vierge.

On ne trouve aucune compilation à la fin de ce premier tome. Le deuxième commence sans présentation et renferme les mots allant de «PON » à "UTO », et ne renferme aucune autre précision.

Les archives $\mathrm{du}$ Scolasticat Saint-Joseph conservent également le manuscrit d'une grammaire montagnaise, de la main du Père de la Brosse, daté de 1768, dont la présentation en latin se traduit ainsi :

Ces éléments de la langue montagnaise à partir des écrits divers des différents missionnaires, qui du moins purent être retrouvés, ont été réunis en un seul volume par Jean-Baptiste de la Brosse, aquitain, prêtre, qui fut le $21^{\circ}$ Père en Dieu et Pasteur des Sauvages montagnais dans les postes du Domaine du Roi.

Oeuvre commencée aux Iles Jérémie, pays des Papinachois, le $16^{\circ}$ jour des calendes de décembre ( 16 novembre), année 1768 , à partir de la maternité de la vierge. 
Cette grammaire compte 29 chapitres et présente les éléments grammaticaux dans l'ordre traditionnel : les lettres, le nom, les adjectifs, etc. Les titres des chapitres sont en latin. A la page 193, on peut lire la remarque suivante :

Outre les notes qui sont relevées ça et là, en supplément, ici on place des remarques du Père Ludovic André et autres sur la langue montagnaise.

Cinquante-ring remarques suivent cet avertissement. Le volume compte environ 201 pages.

Ces manuscrits sont bien conservés. La disposition de la matière et l'écriture témoignent de l'application de l'auteur. Les ratures et les bavures sont à peu près inexistantes et les rares qui s'y trouvent sont très discrètes. Le Père de la Brosse devait avoir une vue très nette de son sujet et un esprit de synthèse peu ordinaire pour en venir à écrire si correctement dès le premier jet.

Soulignons très rapidement, pour terminer cette bibliographie un peu sèche, une œuvre collective, si l'on peut dire, à laquelle le Père de la Brosse a été le dernier à mettre la main, le Miscellaneorum Liber, conservé aux archives de l'archevêché de Québec. C'est le Père de la Brosse qui baptise ainsi le registre dans lequel ses prédécesseurs et lui-même ont inscrit les baptêmes, les mariages et les sépultures. On peut lire également dans ce manuscrit, les annales, en particulier, celles du Père de la Brosse pour les années 1766-1776, ainsi que la liste des bienfaiteurs de la mission. La première page renferme cette note du Père de la Brosse :

L'index de ce Livre a été placé au folio 173 par J. B. de la Brosse, Pasteur de cette mission dans les postes du Domaine du Roi, qui a numéroté chacun des feuillets qu'il y a trouvé afin que grâce à cet index, on retrouve plus facilement ce que l'on cherche.

Au début de l'index même, il écrit: "Index des choses contenues dans cette forêt."

Avant de loger pour de bon aux archives de l'archevêché de Québec, ce manuscrit a connu bien des aventures que le temps ne nous permet pas de décrire ici. Pour satisfaire votre curiosité, nous vous reférons à la Semaine Religieuse de Québec de 1912.

On peut lire encore des actes de baptêmes, de mariages, et de sépultures de la main du Père de la Brosse dans un autre manuscrit, qui a trait aux missions du Saguenay, appelé Magnus Liber, également conservé aux archives de l'archevêché de Québec.

Signalons que nous passons sous silence la correspondance du Père de la Brosse, que nous croyons peu volumineuse, mais non moins très intéressante. 
La besogne abattue par le Père de la Brosse et ses nombreux travaux le prédestinaient à entrer dans la légende. De son vivant, il s'était acquis une réputation de haute sainteté et on lui attribuait même des miracles. Dans les milieux populaires, n'enseigne-t-on pas qu'il avait prédit le jour et l'heure de sa mort et que les cloches avaient sonné d'elles-mêmes, à minuit, pour avertir ses amis que le Père de la Brosse n'était plus de ce monde? Au-delà de tout ce que la légende a pu embellir et amplifier, au sujet du Père de la Brosse, il reste que tout ceci sert à mieux nous faire comprendre l'estime et la vénération que lui témoignaient tous ceux qu'il avait approchés.

"De tous les missionnaires, selon feu J.-Edmond Roy, qui ont exercé l'apostolat sur la terre saguenéenne et dans les régions du Golfe, c'est lui qui a laissé les souvenirs les plus vivaces et les plus vénérés ${ }^{36}$."

\author{
Yves Tremblay, \\ professeur et historien, \\ Québec.
}

36 Société Historique du Saguenay, Dossier 199, p. 17. 